\title{
A group-specific arbitrary tradition in chimpanzees (Pan troglodytes)
}

\author{
Edwin J. C. van Leeuwen - Katherine A. Cronin • \\ Daniel B. M. Haun
}

Received: 12 November 2013/Revised: 8 May 2014/Accepted: 29 May 2014/Published online: 11 June 2014

(c) Springer-Verlag Berlin Heidelberg 2014

\begin{abstract}
Social learning in chimpanzees has been studied extensively and it is now widely accepted that chimpanzees have the capacity to learn from conspecifics through a multitude of mechanisms. Very few studies, however, have documented the existence of spontaneously emerged traditions in chimpanzee communities. While the rigour of experimental studies is helpful to investigate social learning mechanisms, documentation of naturally occurring traditions is necessary to understand the relevance of social learning in the real lives of animals. In this study, we report on chimpanzees spontaneously copying a seemingly nonadaptive behaviour ("grass-in-ear behaviour"). The behaviour entailed chimpanzees selecting a stiff, straw-like blade of grass, inserting the grass into one of their own ears, adjusting the position, and then leaving it in their ear during subsequent activities. Using a daily focal follow procedure, over the course of 1 year, we observed 8 (out of 12) group members engaging in this peculiar behaviour. Importantly, in the three neighbouring groups of chimpanzees $(n=82)$, this behaviour was only observed once, indicating that ecological factors were not determiners of the prevalence of this behaviour. These observations show that chimpanzees
\end{abstract}

Electronic supplementary material The online version of this article (doi:10.1007/s10071-014-0766-8) contains supplementary material, which is available to authorized users.

E. J. C. van Leeuwen $(\square) \cdot$ K. A. Cronin

Max Planck Institute for Psycholinguistics, Max Planck Institute for Evolutionary Anthropology, PO Box 310,

6500 AH Nijmegen, The Netherlands

e-mail: ejcvanleeuwen@gmail.com

D. B. M. Haun

Max Planck Institute for Evolutionary Anthropology, Max Planck Institute for Psycholinguistics, University of Portsmouth, Deutscher Platz 6, 04103 Leipzig, Germany have a tendency to copy each other's behaviour, even when the adaptive value of the behaviour is presumably absent.

Keywords Chimpanzees - Social learning - Chimfunshi Wildlife Orphanage Trust · Arbitrary tradition · Fads

\section{Introduction}

Social learning refers to learning about other agents or the inanimate world that is influenced by observation of, or interaction with, another individual or its products (Heyes 2012). A myriad studies have reported social learning in a variety of species (reviewed in e.g. Galef 2012). In non-human primates, many social learning studies have employed experimental designs using artificial target behaviours. While these experiments effectively explore learning capacities in the absence of confounding variables, the ecological validity of their results is inevitably restricted. To investigate how social learning is actually (rather than plausibly) employed in the lives of animals, documentation of naturally occurring cases is essential.

In addition to tracking the spread of behaviour within a single population, researchers have reasoned about the presence of social learning in non-human animals by reporting on intra-specific between-group differences that are unlikely to have emerged from non-social mechanisms (see Wrangham et al. 1994). This "ethnographic approach" has been criticized for not being able to disentangle the contributions of genetic and ecological factors to the behavioural variation (Laland and Janik 2006). However, when multiple, isolated populations are present in the same ecological environment, this approach gains power. 
In this study, we report on chimpanzees spontaneously copying a seemingly non-adaptive behaviour: sticking grass in one's own ear. Importantly, we present data on four groups of chimpanzees that live in the same forested environment and do not systematically differ by subspecies making the ethnographic approach powerful in this case (see also van Leeuwen et al. 2012).

\section{Materials and methods}

\section{Subjects}

Subjects were 94 chimpanzees across four social groups at the Chimfunshi Wildlife Orphanage Trust, a sanctuary in the north-western part of Zambia (for details, see van Leeuwen et al. 2012). The chimpanzees live in forested enclosures ranging in size from 20 to 80 hectares. Except for Group 3 and Group 4, none of the groups can see each other. The chimpanzees stay outside overnight and only come indoors for supplementary mid-day feeding (11:30-13:30). All groups were formed based on the order of arrival. Only non-infant chimpanzees were included in this study; infants were considered not old enough to copy or engage in the behaviour described in the following section.

\section{The invention}

"Grass-in-ear behaviour" (henceforth "GIEB") was first documented in 2010 when the first author observed one female chimpanzee (Julie) repeatedly putting a stiff, strawlike blade of grass in one or both of her ears. She left the grass hanging out of her ear(s) during subsequent behaviour such as grooming, playing, and resting (Figs. 1, 2 and Online Resource 1); the behaviour served no discernible purpose.

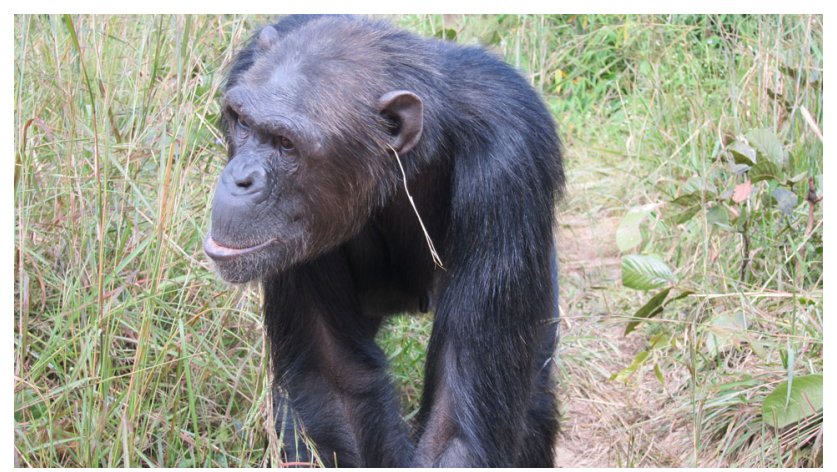

Fig. 1 Julie (the inventor) performing the grass-in-ear behaviour
Data collection and analysis

During subsequent visits to the sanctuary, we observed that GIEB was also shown by other chimpanzees in the same social group. In order to determine whether the GIEB was transmitted via social learning, we analysed 1 year of videos derived from a focal follow procedure that started in February 2011 and yielded $740 \mathrm{~h}$ of video material through February 2012 (Group 1-4: 184, 201, 159 , and $166 \mathrm{~h}$, respectively). Data were collected for $1 \mathrm{~h}$ on each group daily and consisted of ten-minute focal follows centred on a subject that was chosen through systematic sampling of the enclosure. Focal follows included a minimum of 2-metre visibility around the focal subject (for details see van Leeuwen et al. 2012). We operationalized GIEB as "putting grass in one's own ear". In addition to scoring all occurrences of GIEB (scored as present or absent for each individual in each focal follow), we scored whether GIEB occurred simultaneously by more than one individual. In order to test our hypothesis that the GIEB was sparked by social

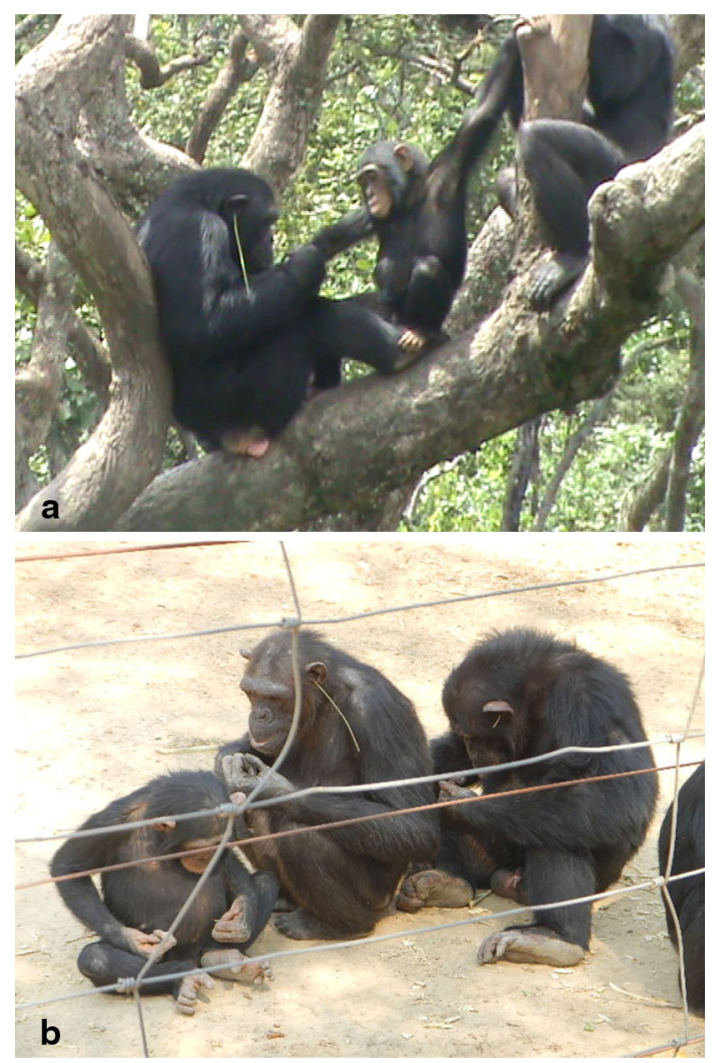

Fig. 2 Grass-in-ear behaviour copied by group members: a Kathy (on the left) and Julie (on the right) are grooming Jack (Julie's son) while having grass hanging out of their ears, b Val (on the right) is grooming Julie (in the middle) while both have grass hanging out of their ears. Jack (on the left) is also visible with a straw of grass in his hands (Photo b taken by Mylène Désilets, used with permission) 
Table 1 GIEB diffused in one of the four neighbouring groups of chimpanzees at the Chimfunshi Wildlife Orphanage Trust: given are the occurrences of GIEB of all individuals in Group 4 as observed in the focal follow videos over the course of February 2011-February 2012

\begin{tabular}{llcc}
\hline & Sex & Age & GIEB (frequency) \\
\hline Julie $^{\mathrm{a}}$ & Female & 18 & 168 \\
Kathy & Female & 13 & 36 \\
Val & Male & 12 & 10 \\
Jack & Male & 4 & 8 \\
Miracle & Female & 11 & 7 \\
Sinkie & Male & 18 & 2 \\
Bobby & Male & 18 & 1 \\
Nicky & Male & 21 & 1 \\
Kambo & Female & 16 & 0 \\
Bertha & Female & 12 & 0 \\
Commander & Male & 12 & 0 \\
Kit & Male & 7 & 0 \\
\hline
\end{tabular}

${ }^{a}$ Inventor of the GIEB

processes rather than by repeated individual inventions, we analysed the likelihood of the observed distribution of GIEB over all subjects across the four social groups given a randomly assigned distribution using a two-sided Fisher's exact test with simulated $P$ value (Monte Carlo randomizations). Additionally, the frequency of GIEB co-occurrences was investigated to i) analyse whether social learning could have taken place (i.e. social learning requires proximity) and ii) to possibly shed light on the learning mechanism(s) involved. After the GIEB inventor died on 22 May 2013, we analysed all available Group 4 data collected afterwards (up to 14 July 2013, yielding $25 \mathrm{~h}$ of video material) in order to investigate whether the GIEB outlasted its inventor.

\section{Results}

GIEB was only observed in one of the four chimpanzee groups (Group 4), with the exception of one occurrence in one other group (Group 2). In total, 8 of 12 chimpanzees of Group 4 were observed to (repeatedly) engage in GIEB by February 2012 (Table 1: also see Fig. 3 and Online Resource 1-3 for example videos). The observed distribution of GIEB subjects over the four social groups was unlikely to be random (Fisher's exact test: $P<0.0005$ ). In Group 4, almost all GIEB of the imitators was observed simultaneously with the GIEB of at least one other individual, the GIEB inventor $(61 / 65=93.8 \%$ of the cases $)$. Finally, two individuals (Kathy and Val) were observed to engage in GIEB after the inventor had died ( 2 and 5 times, respectively), none of those times simultaneously. Up to the final writing of this communication (28 April 2014), both Kathy and Val have been engaging in GIEB (pers. comm., Innocent Chitalu Mulenga, manager at Chimfunshi).

\section{Discussion}

This study reports the existence of a non-adaptive behavioural tradition in untrained chimpanzees. The behaviour consisted of putting a straw of grass in one's own ear, and while it occurred frequently and by the majority of individuals in one group, it was only observed once across the three other groups of chimpanzees. Since the chimpanzee groups at Chimfunshi live in one continuous woodland and do not systematically differ by subspecies, it is difficult to imagine that ecological and/or genetic factors caused the disproportionate concentration of GIEB individuals in one group. Further taking into account that most GIEB occurred in proximity to another individual engaging in the GIEB, it seems parsimonious to interpret the observations in terms of a socially learned behavioural pattern.

In a recent study investigating the strength of associations within and between groups (Cronin et al. 2014), where association is defined as frequency with which individuals are observed in proximity to one another, Julie's highest association score was with her son, Jack, who was the first chimpanzee observed to acquire the GIEB. She also associated more with Kathy, the second chimpanzee observed to acquire the behaviour, than she did on average with other chimpanzees in the group. The next two imitators, Miracle and Val, respectively, had average association scores with Julie, but approached Julie more frequently than the average approach tendency in the group. These data indicate a relationship between proximity and social learning as has been seen in other research (e.g. Bonnie and De Waal 2006), but given the nature of the behaviour and the observational schedule, we cannot establish a definite link.

Natural observations like the ones described in the present report are important because they show that social learning occurs spontaneously amongst chimpanzees (as opposed to experimentally imposed/rewarded; also see Whiten et al. 1999). As such, the GIEB may be reminiscent of chimpanzees' tool-use acquisition (e.g. Luncz et al. 2012) or grooming handclasp behaviour (e.g. van Leeuwen et al. 2012). However, the GIEB observations may be additionally informative because the chimpanzees adopted a behaviour that is not an obviously functional manipulation of the physical world (as in the tool-use context) or the social environment (as in the grooming handclasp context). Due to its spontaneous and arbitrary nature, the GIEB may be most comparable to the observation that able-bodied 


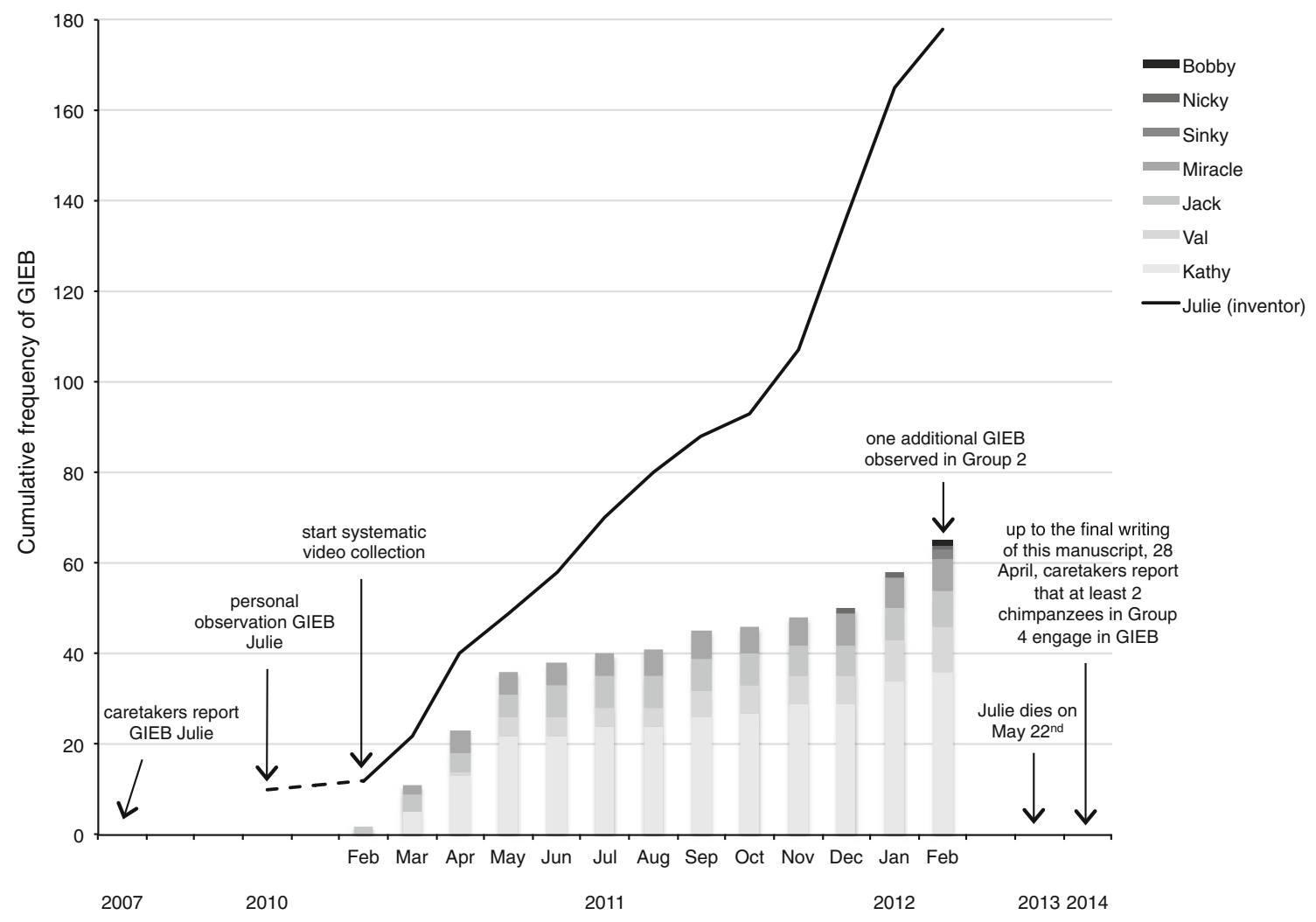

Fig. 3 Cumulative record of GIEB in Group 4 at the Chimfunshi Wildlife Orphanage Trust for Julie the inventor (black line) and the imitators (stacked bars) separately. Julie was reported to engage in GIEB in 2007 by the chimpanzee caretakers, which was verified by informal observation of at least ten independent GIEB bouts by the first author in 2010. No other chimpanzees were reported or observed to engage in GIEB at that time. After Julie's death in May 2013, seven
GIEB bouts (two individuals) have been observed during systematic analysis of additional video material up to 14 July 2013 (see "Materials and methods" and "Results"). By the time of the final writing of this manuscript, 28 April 2014, the same two chimpanzees as were observed in the videos taken between 22 May and 14 July (Kathy and Val) still engage in GIEB chimpanzees copied a motor procedure from a chimpanzee who had been handicapped by a snare trap and was therefore forced to scratch his back in an unorthodox way (Hobaiter and Byrne 2010). Those authors concluded that because the able-bodied chimpanzees could scratch themselves in chimpanzee-typical ways, they copied an "unnecessary behavioural trait", which was interpreted in terms of (program-level) imitation (Hobaiter and Byrne 2010). The GIEB may be best explained by lower-level mechanisms. As the GIEB almost exclusively occurred simultaneously, response facilitation seems a likely mechanistic explanation. Although response facilitation commonly refers to triggering familiar behaviour (Rendell et al. 2011), the novel sequence of putting grass in one's own ear may well fit the scope of this mechanism (Byrne and Russon 1998). The observation that GIEB was also observed in isolation may indicate that while response facilitation had broadened the behavioural repertoires of the imitators, only some experienced the behaviour as somehow rewarding and continued engaging in it on their own (Galef 1995). Finally, in conjunction with its arbitrary nature, the perpetuation of socially learned behaviour in the absence of the original inventor, despite its low frequency, links the GIEB observations to human cultural phenomena (Boesch 2013).

Regardless of the precise mechanism underlying the behavioural diffusion, our observations importantly show that chimpanzees spontaneously copy arbitrary behaviour from their group members. In line with Hobaiter and Byrne (2010), we interpret our data as reflecting chimpanzees' proclivity to actively investigate and learn from group members' behaviours in order to obtain biologically relevant information. The fact that these behaviours can be arbitrary and outlast the originator speaks to the cultural potential of chimpanzees.

Acknowledgments Dedicated to Julie and her son Jewel. The authors are grateful to the Zambia Wildlife Authority and the Chimfunshi Wildlife Orphanage Trust for hosting this project. Many thanks to Innocent Chitalu Mulenga (CWOT manager), the Chimfunshi staff (Patrick Chambatu, Thomson Mbilishi, Albert Mulembo, 
Goodson Muletele, Felix Chinyama, Patrick Mwika, Mumba Kawele, Misheck Kasongo, John Kayuya and Joseph Kasongo), Ian Ferreira, Mylène Désilets, and the video coding team including Vivian Vreeman, Marjolein van Ginneken, Karoline Kneist, Rebecca Koomen, and Maddalena Tacchetti.

\section{References}

Boesch C (2013) Wild cultures: a comparison between chimpanzee and human cultures. Cambridge University Press, Cambridge

Bonnie KE, De Waal FBM (2006) Affiliation promotes the transmission of a social custom: handclasp grooming among captive chimpanzees. Primates 47:27-34. doi:10.1007/s10329005-0141-0

Byrne RW, Russon AE (1998) Learning by imitation: a hierarchical approach. Behav Brain Sci 21(5):667-721

Cronin KAC, Van Leeuwen EJC, Vreeman V, Haun DBM (2014) Population-level variability in the social climates of four chimpanzee societies. Evol Hum Behav. doi:10.1016/j.evolhum behav.2014.05.004

Galef BG (1995) Why behavior patterns that animals learn socially are locally adaptive. Anim Behav 49(5):1325-1334. doi:10. 1006/Anbe.1995.0164

Galef BG (2012) Social learning and traditions in animals: evidence, definitions, and relationship to human culture. Wires Cogn Sci 3(6):581-592. doi:10.1002/Wcs.1196
Heyes C (2012) What's social about social learning? J Comp Psychol 126(2):193-202. doi:10.1037/A0025180

Hobaiter C, Byrne RW (2010) Able-bodied wild chimpanzees imitate a motor procedure used by a disabled individual to overcome handicap. PLoS ONE 5(8):e11959. doi:10.1371/journal.pone. 0011959

Laland KN, Janik VM (2006) The animal cultures debate. Trends Ecol Evol 21(10):542-547. doi:10.1016/J.Tree.06.005

Luncz LV, Mundry R, Boesch C (2012) Evidence for cultural differences between neighboring chimpanzee communities. Curr Biol 22(10):922-926. doi:10.1016/J.Cub.03.031

Rendell L, Fogarty L, Hoppitt WJE, Morgan TJH, Webster MM, Laland KN (2011) Cognitive culture: theoretical and empirical insights into social learning strategies. Trends Cogn Sci 15(2):68-76

van Leeuwen EJC, Cronin KAC, Haun DBM, Mundry R, Bodamer MD (2012) Neighbouring chimpanzee communities show different preferences in social grooming behaviour. Proc R Soc B-Biol Sci 279:4362-4367. doi:10.1098/rspb.2012.1543

Whiten A, Goodall J, McGrew WC, Nishida T, Reynolds V, Sugiyama Y, Tutin CEG, Wrangham RW, Boesch C (1999) Cultures in chimpanzees. Nature 399(6737):682-685. doi:10. $1038 / 21415$

Wrangham RW, de Waal FBM, McGrew WC (1994) The challenge of behavioral diversity. In: Wrangham RW, McGrew WC, de Waal FBM, Heltne PG (eds) Chimpanzee cultures. Harvard University Press, Cambridge, pp 1-18 